TITLE:

\title{
Shortest bibranchings and valuated matroid intersection
}

\author{
$\operatorname{AUTHOR}(\mathrm{S}):$ \\ Takazawa, Kenjiro
}

CITATION:

Takazawa, Kenjiro. Shortest bibranchings and valuated matroid intersection. Japan Journal of Industrial and Applied Mathematics 2012, 29(3): 561-573

ISSUE DATE:

2012-10

URL:

http://hdl.handle.net/2433/167968

\section{RIGHT:}

The final publication is available at www.springerlink.com; この論文は 出版社版でありません。引用の際には出版社版をご確認ご利用くださ $\omega_{\circ}$; This is not the published version. Please cite only the published version. 


\title{
A weighted independent even factor algorithm
}

\author{
Kenjiro Takazawa \\ Research Institute for Mathematical Sciences, \\ Kyoto University, Kyoto 606-8502, Japan. \\ takazawa@kurims.kyoto-u.ac.jp
}

\begin{abstract}
For a digraph $D=(V, A)$ and a partition $\{S, T\}$ of $V$, an arc set $B \subseteq A$ is called an $S$ $T$ bibranching if each vertex in $T$ is reachable from $S$ and each vertex in $S$ reaches $T$ in the subgraph $(V, B)$. Bibranchings commonly generalize bipartite edge covers and arborescences. A totally dual integral linear system determining the $S-T$ bibranching polytope is provided by Schrijver, and the shortest $S-T$ bibranching problem, whose objective is to find an $S-T$ bibranching of minimum total arc weight, can be solved in polynomial time by the ellipsoid method or a faster combinatorial algorithm due to Keijsper and Pendavingh.

The valuated matroid intersection problem, introduced by Murota, is a weighted generalization of the independent matching problem, including the independent assignment problem and the weighted matroid intersection problem. The valuated matroid intersection problem can be solved efficiently with polynomially many value oracles by extending classical combinatorial algorithms for the weighted matroid intersection problem.

In this paper, we show that the shortest $S$ - $T$ bibranching problem is polynomially reducible to the valuated matroid intersection problem. This reduction suggests one answer to why the shortest $S$-T bibranching problem is tractable, and implies new combinatorial algorithms for the shortest $S$ - $T$ bibranching problem based on the valuated matroid intersection algorithm, where a value oracle corresponds to computing a minimum-weight arborescence.
\end{abstract}

Keywords: Shortest bibranching, Arborescence, Valuated matroid intersection, Discrete convex function

\section{Introduction}

Let $D=(V, A)$ be a directed graph (digraph) with vertex set $V$ and arc set $A$, and let $w \in \mathbf{R}_{+}^{A}$ be a nonnegative arc-weight vector. For a partition $\{S, T\}$ of $V$, an $\operatorname{arc}$ subset $B \subseteq A$ is called an $S$-T bibranching if each vertex in $T$ is reachable from $S$ and each vertex in $S$ reaches $T$ in the subgraph $(V, B)$.

The notion of bibranchings was introduced by Schrijver [17]. Bibranchings commonly generalize bipartite edge covers and arborescences.

Bipartite edge cover. In an undirected graph $G=(V, E)$, an edge set $F \subseteq E$ is called an edge cover if every vertex is incident to at least one edge in $F$.

Suppose that $G$ is a bipartite graph with color classes $S$ and $T$. Then, obtain a digraph $D$ from $G$ by orienting every edge toward $T$. It is straightforward to see that an edge subset in $G$ is an edge cover if and only if its corresponding arc subset in $D$ is an $S$ - $T$ bibranching. 
Arborecsence. For a digraph $D=(V, A)$ and a vertex $r \in V$, an $\operatorname{arc}$ set $F \subseteq A$ is an $r$ arborescence if $F$ contains no undirected cycle and each vertex $v \in V \backslash\{r\}$ has exactly one entering edge in $F$ while $r$ has no enterng edge in $F$. Here, consider a partition $\{S, T\}$ of $V$, where $S=\{r\}$ and $T=V \backslash\{r\}$. Then, it is easy to see that a minimal $S$-T bibranching is an $r$-arborescence.

A typical optimization problem concerning edge covers and arborescences is to find those of minimum weight, when a non-negative edge/arc weight is given. So, our main interest is in the shortest $S-T$ bibranching problem, the objective of which is to find an $S$ - $T$ bibranching $B$ minimizing $w(B)=\sum_{e \in B} w(e)$. Generally, for a finite set $V$ and a vector $x \in \mathbf{R}^{V}$, we denote $x(X)=\sum_{v \in X} x(v)$ for $X \subseteq V$.

A min-max relation for the minimum size of an $S-T$ bibranching follows from the KönigRado edge cover theorem. Furthermore, Schrijver [17] presented a totally dual integral linear system determining the $S$-T bibranching polytope (see also Schrijver [19]). The shortest $S-T$ bibranching problem can be solved in polynomial time by the ellipsoid method [17], and Keijsper and Pendavingh [9] designed a combinatorial primal-dual algorithm with running time $\mathrm{O}\left(n^{\prime}(m+\right.$ $n \log n)$ ), where $n^{\prime}=\min \{|S|,|T|\}, n=|S \cup T|$ and $m=|A|$. This time complexity is almost best possible, since the current best time complexity for the minimum-weight edge cover problem is $\mathrm{O}\left(n^{\prime}(m+n \log n)\right)[3,24]$ and that for the minimum-weight arborescence problem is $\mathrm{O}(m+n \log n)$ [7]. Therefore, it could be understood that the shortest $S$ - $T$ bibranching problem is as tractable as its special cases of the minimum-weight edge cover and minimum-weight arborescence problems, and that the tractability derives from the total dual integrality of the linear system determining the $S-T$ bibranching polytope.

In this paper, we exhibit a new feature of the shortest $S$ - $T$ bibranching problem which explains its tractability: the shortest $S-T$ bibranching problem is a special case of the valuated matroid intersection problem, introduced by Murota [12,13]. For a function $\omega: 2^{V} \rightarrow \mathbf{R} \cup\{-\infty\}$, let $\operatorname{dom} \omega$ denote the effective domain of $\omega$, which is defined by $\operatorname{dom} \omega=\{X \mid X \subseteq V, \omega(X)>-\infty\}$. A function $\omega: 2^{V} \rightarrow \mathbf{R} \cup\{-\infty\}$ with $\operatorname{dom} \omega \neq \emptyset$ is called a valuated matroid [1,2] if it satisfies the following exchange property:

(VM) for each $X, Y \in 2^{V}$ and $u \in X \backslash Y$, there exists $v \in Y \backslash X$ such that

$$
\omega((X \backslash\{u\}) \cup\{v\})+\omega((Y \backslash\{v\}) \cup\{u\}) \geq \omega(X)+\omega(Y) .
$$

It is easy to see that $\operatorname{dom} \omega$ forms the base family of a matroid of ground set $V$. The valuated matroid intersection problem is described as follows.

\section{The valuated matroid intersection problem}

Instance: $\bullet$ a ground set $V$,

- a weight vector $w \in \mathbf{R}^{V}$, and

- two valuated matroids $\omega^{+}: 2^{V} \rightarrow \mathbf{R} \cup\{-\infty\}$ and $\omega^{-}: 2^{V} \rightarrow \mathbf{R} \cup\{-\infty\}$.

Objective: maximize $w(X)+\omega^{+}(X)+\omega^{-}(X)$.

The valuated matroid intersection problem is a special case of the valuated independent assignment problem, which is a weighted generalization of the independent matching problem, and includes other important problems such as the independent assignment problem [8] and the weighted matroid intersection problem. The valuated independent assignment problem can be solved efficiently 
with polynomially many value oracles: Murota [13] designed combinatorial algorithms by naturally extending the classical weighted matroid intersection algorithms $[4,8,10]$. For more information on the valuated matroid intersection problem, readers are referred to Murota [15].

Our main result shows a relation between the shortest bibranching and valuated matroid intersection problems.

Theorem 1. The shortest $S-T$ bibranching problem is polynomially reducible to the valuated matroid intersection problem.

Theorem 1 reveals a new feature of bibranchings underlying the tractability of the shortest $S$ - $T$ bibranching problem. Furthermore, by combining this reduction and the valuated independent assignment algorithms in [13], we obtain new combinatorial algorithms for the shortest $S-T$ bibranching problem which is based on the weighted matroid intersection algorithms.

In proving Theorem 1, we make use of theory on a more general framework of discrete convex analysis [14], in particular theory on $\mathrm{M}$-convex and $\mathrm{M}^{\natural}$-convex functions $[11,16]$ (see also [6]). Our argument is an example where theory of a generalized framework gives a new insight of a certain special case.

The organization of this paper is as follows. In Section 2, we review bibranchings, valuated matroids, and M-convex and $\mathrm{M}^{\natural}$-convex functions. Section 3 is devoted to proving Theorem 1 . In Section 4 , we conclude this paper with a few remarks.

\section{Preliminaries}

\subsection{Digraphs}

Let $D=(V, A)$ be a digraph. An arc from $u$ to $v$ is denoted by $u v$. For an arc $a$, the initial vertex is denoted by $\partial^{+} a$ and the terminal vertex is denoted by $\partial^{-} a$. For a vertex $v \in V$, the set of arcs with tail $v$ is denoted by $\delta^{+} v$, and those with head $v$ is denoted by $\delta^{-} v$. For $X \subseteq V$, let $D[X]=(U, A[X])$ denote the subgraph induced by $X$, i.e., $A[X]=\left\{a \mid a \in A, \partial^{+} a \in X, \partial^{-} a \in X\right\}$. For $X, Y \subseteq V$, let $A[X, Y]=\left\{a \mid a \in A, \partial^{+} a \in X, \partial^{-} a \in Y\right\}$.

A path in a digraph is a sequence $\left(v_{0}, a_{1}, v_{1}, \ldots, v_{k-1}, a_{k}, v_{k}\right)$, where $a_{i}=v_{i-1} v_{i}$ for every $i=1, \ldots, k$ and $v_{0}, v_{1}, \ldots, v_{k}$ are distinct. A cycle is a sequence $\left(v_{0}, a_{1}, v_{1}, \ldots, v_{k-1}, a_{k}, v_{k}\right)$, where $a_{i}=v_{i-1} v_{i}(i=1, \ldots, k), v_{0}, v_{1}, \ldots, v_{k-1}$ are distinct, and $v_{0}=v_{k}$. For $v \in V$ and $U \subseteq V$, we say that $v$ reaches $U$ if there exists a path starting from $v$ and ending in some vertex in $U$, and that $v$ is reachable from $U$ if there exists a path starting from some vertex in $U$ and ending in $v$.

For an arc set $F \subseteq A$, its reversal is an arc set obtained by reversing every arc in $F$, that is, $\{v u \mid u v \in F\}$. For a digraph $D=(V, A)$, an arc subset $B \subseteq A$ is a branching if $\left|B \cap \delta^{-} v\right| \leq 1$ for every $v \in V$ and $B$ contains no cycle. A cobranching is a set of edges whose reversal is a branching.

For an arc subset $B \subseteq A$, define $\partial^{+} B=\bigcup_{a \in B} \partial^{+} a$ and $\partial^{-} B=\bigcup_{a \in B} \partial^{-} a$. If $B$ is a branching, a vertex in $V \backslash \partial^{-} B$ is called a root of $B$ and we denote the set of roots by $R(B)$, i.e., $R(B)=V \backslash \partial^{-} B$. Similarly, for a cobranching $B^{\prime}$, we define $R^{*}\left(B^{\prime}\right)=V \backslash \partial^{+} B^{\prime}$. Recall that, for a vertex $r \in V$, an $r$-arborescence is a branching $B$ with $R(B)=\{r\}$.

Let $D=(V, A)$ be a digraph and let $\{S, T\}$ be a partition of $V$. That is, $S \cap T=\emptyset$ and $S \cup T=V$. Recall that an arc set $B \subseteq A$ is an $S-T$ bibranching if, in the subgraph $(V, B)$, every vertex in $T$ is reachable from $S$ and every vertex in $S$ reaches $T$. A set of $\operatorname{arcs} C \subseteq A$ is called an $S-T$ bicut if $C=A[V \backslash U, U]=\left\{a \mid \partial^{+} a \in V \backslash U, \partial^{-} a \in U\right\}$ for some nonempty proper subset $U$ of $V$ such that $U \subseteq T$ or $U \supseteq T$. 
The following linear system describes the $S$ - $T$ bibranching polytope:

$$
\begin{array}{ll}
0 \leq x(a) \leq 1 & \text { for each } a \in A, \\
x(C) \geq 1 & \text { for each } S-T \text { bicut } C .
\end{array}
$$

Theorem 2 (Schrijver [17]). The linear system consisting of (1) and (2) is totally dual integral.

\subsection{Valuated matroids, $M$-convex functions and $M^{\natural}$-convex functions}

In this subsection, beginning with valuated matroids, we exhibit several basic facts on $M$-convex functions [11] and $M^{\natural}$-convex functions [16], which provide broader frameworks than that of valuated matroids, and also their relation to minimum-weight branchings. For more detailed information on these discrete convex functions, readers are referred to Murota [14] and Fujishige [6].

Let us begin with matroids. Let $V$ be a finite set and $\mathcal{B} \subseteq 2^{V}$ be its subset family with $\mathcal{B} \neq \emptyset$. Then, we say that $(V, \mathcal{B})$ is a matroid if, for each $X, Y \in \mathcal{B}$ and $u \in X \backslash Y$, there exists $v \in Y \backslash X$ such that $(X \backslash\{u\}) \cup\{v\} \in \mathcal{B}$.

Actually, this exchange axiom is equivalent to the following simultaneous exchange property:

(M) for each $X, Y \in \mathcal{B}$ and $u \in X \backslash Y$, there exists $v \in Y \backslash X$ such that

$$
(X \backslash\{u\}) \cup\{v\} \in \mathcal{B} \quad \text { and } \quad(Y \cup\{u\}) \backslash\{v\} \in \mathcal{B} .
$$

By considering this simultaneous exchange property, we can define a quantitative generalization (VM) in Section 1, which defines valuated matroids.

Matroids are defined as a pair of a ground set $V$ and the base family $\mathcal{B} \subseteq 2^{V}$. By identifying a subset of $V$ as a vector in $\{0,1\}^{V}, \mathcal{B}$ could be seen as a subfamily of $\{0,1\}^{V}$. M-convex sets offer a generalization of matroids which is defined as a subfamily of $\mathbf{Z}^{V}$.

For $X \subseteq V$, the characteristic vector of $X$ is a vector $\chi_{X} \in \mathbf{Z}^{V}$ defined by $\chi_{X}(v)=1$ for $v \in X$ and $\chi_{X}(v)=0$ for $v \in V \backslash X$. For an element $u \in V, \chi_{\{u\}}$ is abbreviated as $\chi_{u}$. For a vector $x \in \mathbf{Z}^{V}$, define $\operatorname{supp}^{+}(x)=\{v \mid v \in V, x(v)>0\}$ and $\operatorname{supp}^{-}(x)=\{v \mid v \in V, x(v)<0\}$

A set of integer points $B \subseteq \mathbf{Z}^{V}$ is an $M$-convex set if it satisfies the following exchange axiom:

(B-EXC) for each $x, y \in B$ and $u \in \operatorname{supp}^{+}(x-y)$, there exists $v \in \operatorname{supp}^{-}(x-y)$ such that

$$
x-\chi_{u}+\chi_{v} \in B \text { and } y+\chi_{u}-\chi_{v} \in B .
$$

Note that (B-EXC) generalizes the axiom (M). Now, we can define M-convex functions by generalizing (DM). For a function $f: \mathbf{Z}^{V} \rightarrow \mathbf{R} \cup\{+\infty\}$, let $\operatorname{dom} f=\{x \mid f(x)<+\infty\}$. A function $f: \mathbf{Z}^{V} \rightarrow \mathbf{R} \cup\{+\infty\}$ with $\operatorname{dom} f \neq \emptyset$ is called an $M$-convex function if it satisfies the following property:

(M-EXC) for each $x, y \in \mathbf{Z}^{V}$ and $u \in \operatorname{supp}^{+}(x-y)$, there exists $v \in \operatorname{supp}^{-}(x-y)$ such that

$$
f\left(x-\chi_{u}+\chi_{v}\right)+f\left(y+\chi_{u}-\chi_{v}\right) \leq f(x)+f(y) .
$$

A function $g$ is said to be $M$-concave if $-g$ is M-convex. It is straightforward to see that a valuated matroid is exactly an M-concave function $g$ with dom $g \subseteq\{0,1\}^{V}$.

We can also consider slightly weaker exchange properties. A set $Q \subseteq \mathbf{Z}^{V}$ is an $M^{\natural}$-convex set if it satisfies the following exchange axiom: 
(B'-EXC) for each $x, y \in Q$ and $u \in \operatorname{supp}^{+}(x-y)$, it holds that

$$
x-\chi_{u} \in Q \text { and } y+\chi_{u} \in Q,
$$

or there exists $v \in \operatorname{supp}^{-}(x-y)$ such that

$$
x-\chi_{u}+\chi_{v} \in Q \quad \text { and } \quad y+\chi_{u}-\chi_{v} \in Q .
$$

It is known that an $\mathrm{M}^{\natural}$-convex set is the set of integer vectors in an integral generalized polymatroid $[5,23]$ (see also $[6,14]$ ). Thus, we often refer to a pair $(V, \mathcal{F})$, where $\mathcal{F} \subseteq 2^{V}$, as a generalized matroid if (B⿱ -EXC) holds for $Q=\left\{\chi_{X} \mid X \in \mathcal{F}\right\} \subseteq\{0,1\}^{V}$.

A function $f: \mathbf{Z}^{V} \rightarrow \mathbf{R} \cup\{+\infty\}$ with $\operatorname{dom} f \neq \emptyset$ is called an $M^{\natural}$-convex function if it satisfies the following property:

(M-EXC) for each $x, y \in \mathbf{Z}^{V}$ and $u \in \operatorname{supp}^{+}(x-y)$, it holds that

$$
f\left(x-\chi_{u}\right)+f\left(y+\chi_{u}\right) \leq f(x)+f(y),
$$

or there exists $v \in \operatorname{supp}^{-}(x-y)$ such that

$$
f\left(x-\chi_{u}+\chi_{v}\right)+f\left(y+\chi_{u}-\chi_{v}\right) \leq f(x)+f(y) .
$$

A function $g$ is said to be $M^{\natural}$-concave if $-g$ is $\mathrm{M}^{\natural}$-convex.

The class of $\mathrm{M}^{\natural}$-convex functions is indeed a proper generalization of M-convex functions. On the other hand, in the following sense these two are essentially equivalent. Let $v_{0}$ be an element distinct from $V$ and denote $\tilde{V}=\left\{v_{0}\right\} \cup V$. Let $\alpha$ be an integer. For a function $f: \mathbf{Z}^{V} \rightarrow \mathbf{R} \cup\{+\infty\}$, define $\tilde{f}: \mathbf{Z}^{\tilde{V}} \rightarrow \mathbf{R} \cup\{+\infty\}$ by

$$
\tilde{f}\left(x_{0}, x\right)=\left\{\begin{array}{ll}
f(x) & \text { if } x(\tilde{V})=\alpha, \\
+\infty & \text { otherwise }
\end{array} \quad\left(x_{0} \in \mathbf{Z}, x \in \mathbf{Z}^{V}\right) .\right.
$$

Murota and Shioura [16] proved the following relation between M-convexity and $\mathrm{M}^{\natural}$-convexity.

Theorem 3 ([16]). A function $f: \mathbf{Z}^{V} \rightarrow \mathbf{R} \cup\{+\infty\}$ is an $M^{\natural}$-convex function if and only if $\tilde{f}$ defined by (3) is an M-convex function.

An important operation on M-convex ( $\mathrm{M}^{\natural}$-convex) functions is transformation through a network. Let $(N, E)$ be a digraph with entrance set $N_{1} \subseteq N$ and exit set $N_{2} \subseteq N$, where $N_{1}$ and $N_{2}$ are disjoint. Let $\underline{c}: E \rightarrow \mathbf{Z} \cup\{-\infty\}$ and $\bar{c}: E \rightarrow \mathbf{Z} \cup\{+\infty\}$ denote the lower and upper capacity functions, respectively. For each arc $e \in E$, a cost function $\gamma_{e}: \mathbf{Z} \rightarrow \mathbf{R} \cup\{+\infty\}$ is attached. We assume that $\gamma_{e}$ is a univariate convex function for every $e \in E$. For a flow $\xi \in \mathbf{Z}^{E}$, define its boundary $\partial \xi \in \mathbf{Z}^{N}$ by $\partial \xi(v)=\xi\left(\delta^{+} v\right)-\xi\left(\delta^{-} v\right)$.

Given a function $f_{1}: \mathbf{Z}^{N_{1}} \rightarrow \mathbf{R} \cup\{+\infty\}$, define a function $f_{2}: \mathbf{Z}^{N_{2}} \rightarrow \mathbf{R} \cup\{ \pm \infty\}$ by

$$
\begin{aligned}
f_{2}(y)=\inf _{x, \xi}\left\{f_{1}(x)+\sum_{e \in E} \gamma_{e}(\xi(e)) \mid \xi \in \mathbf{Z}^{E}, \underline{c}(e) \leq \xi(e) \leq \bar{c}(e) \forall e \in E,\right. \\
\left.\partial \xi=(x,-y, 0),(x,-y, 0) \in \mathbf{Z}^{N_{1}} \times \mathbf{Z}^{N_{2}} \times \mathbf{Z}^{N \backslash\left(N_{1} \cup N_{2}\right)}\right\} .
\end{aligned}
$$

We have that $\mathrm{M}$-convexity and $\mathrm{M}^{\natural}$-convexity are maintained in this transformation. 
Theorem 4 ([11, 20]). Assume that $f_{2}$ defined in (4) satisfies $f_{2}>-\infty$. It holds that

- if $f_{1}$ is an $M$-convex function, then $f_{2}$ is also an $M$-convex function; and

- if $f_{1}$ is an $M^{\natural}$-convex function, then $f_{2}$ is also an $M^{\natural}$-convex function.

If an $\mathrm{M}^{\natural}$-convex function $f$ satisfies that $\operatorname{dom} f \subseteq\{0,1\}^{V}$, then $f$ could be seen as a valuation on a generalized matroid by corresponding a subset $X \subseteq V$ to a vector $\chi_{X} \in\{0,1\}$. That is, we can define a function $g: 2^{V} \rightarrow \mathbf{R} \cup\{+\infty\}$ by

$$
g(X)=f\left(\chi_{X}\right) \quad(X \subseteq V) .
$$

We refer to such a set function $g$ obtained from an $\mathrm{M}^{\natural}$-convex function $f$ with $\operatorname{dom} f \subseteq\{0,1\}^{V}$ as an $\mathrm{M}^{\natural}$-convex function, as well.

An example of such an $\mathrm{M}^{\natural}$-convex function arises from branchings. For a weighted digraph $(D, w)$ of $D=(V, A)$ and $w \in \mathbf{R}^{A}$, define $\mathcal{F} \subseteq 2^{V}$ by

$$
\mathcal{F}=\{R(B) \mid B \subseteq A \text { is a branching in } G\} .
$$

Further, define a function $f: 2^{V} \rightarrow \mathbf{R} \cup\{+\infty\}$ by

$$
f(X)= \begin{cases}\min \{w(B) \mid B \text { is a branching, } R(B)=X\} & \text { if } X \in \mathcal{F}, \\ +\infty & \text { if } X \notin \mathcal{F} .\end{cases}
$$

In [22], it is declared that the following theorems follow from arguments in Schrijver [18].

Theorem 5 ([22]). For a digraph $D=(V, A)$, the pair $(V, \mathcal{F})$ defined by $(5)$ is a generalized matroid.

Theorem 6 ([22]). For a weighted digraph $(D, w)$ of $D=(V, A)$ and $w \in \mathbf{R}^{A}$, the function $f$ defined by (6) is an $M^{\natural}$-convex function.

Note that $\mathcal{F}$ is not an M-convex set, and $f$ is not an M-convex function.

Their cobranching counterparts $\mathcal{F}^{*} \subseteq 2^{V}$ and $f^{*}: 2^{V} \rightarrow \mathbf{R} \cup\{+\infty\}$ are defined as follows:

$$
\begin{aligned}
& \mathcal{F}^{*}=\left\{R^{*}(B) \mid B \subseteq A \text { is a cobranching in } G\right\}, \\
& f^{*}(X)= \begin{cases}\min \left\{w(B) \mid B \text { is a cobranching, } R^{*}(B)=X\right\} & \text { if } X \in \mathcal{F}^{*}, \\
+\infty & \text { if } X \notin \mathcal{F}^{*}\end{cases}
\end{aligned}
$$

It is straightforward to see that $\left(V, \mathcal{F}^{*}\right)$ is a generalized matroid and $f^{*}$ is an $\mathrm{M}^{\natural}$-convex funtion.

We summarize the relation among the notions mentioned in this subsection in Figure 1.

\section{Reduction of shortest bibranching to valuated matroid intersec- tion}

In this section, we prove Theorem 1 by exhibiting a polynomial reduction of the shortest $S-T$ bibranching problem to the valuated matroid intersection problem. 


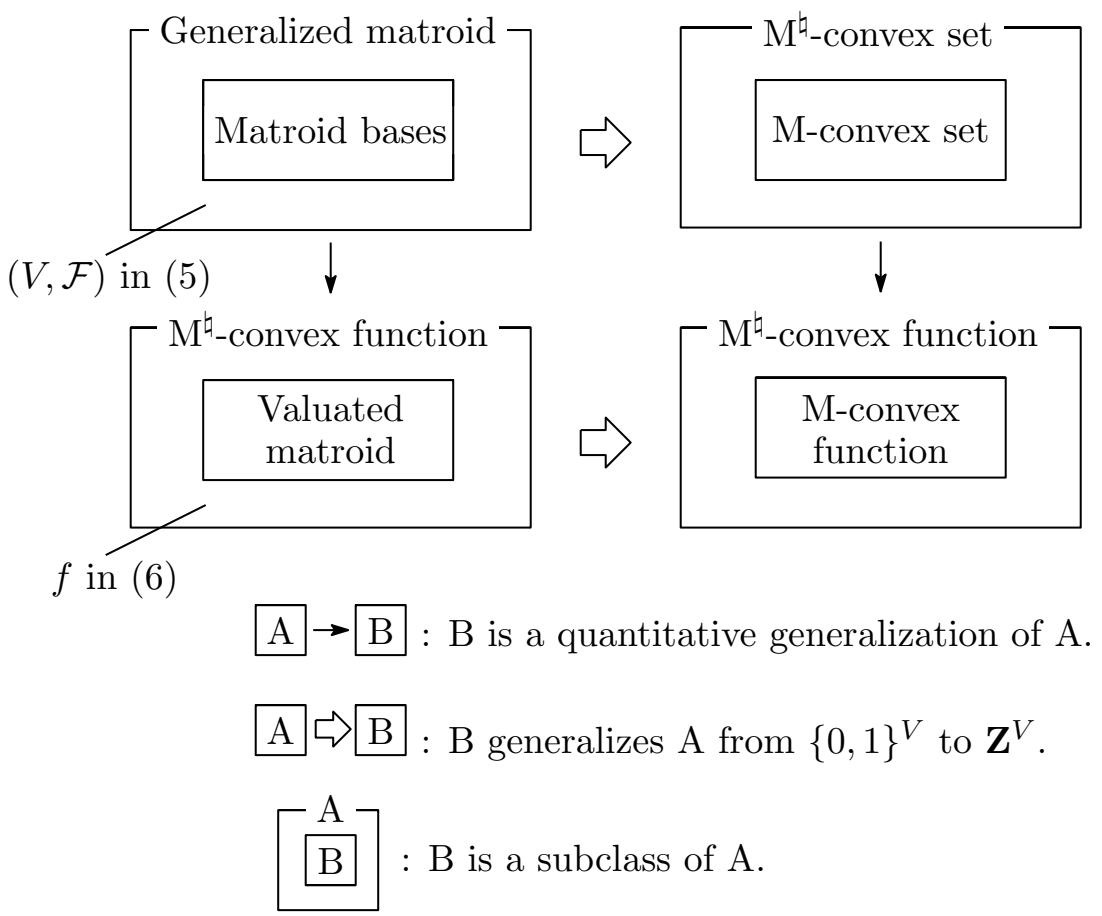

Figure 1: Relation among the notions.

\subsection{A matroidal formulation of the shortest bibranching problem}

Let $(D, w)$ be a weighted digraph with $D=(V, A)$ and $w \in \mathbf{R}_{+}^{A}$, and let $\{S, T\}$ be a partition of $V$. As stated in Section 2.1, the shortest $S-T$ bibranching problem is exactly finding an intger vector $x \in \mathbf{R}^{A}$ minimizing $\sum_{a \in A} w(a) x(a)$ under the constraints (1) and (2), which is tractable by the fact that the system defined by (1) and (2) is totally dual integral (Theorem 2).

Here, we present another formulation of the shortest $S-T$ bibranching problem focusing on the matroidal structure of branchings and cobranchings (Theorems 5 and 6 ). In $D[S]$, define $\mathcal{F}_{S}^{*} \subseteq 2^{S}$ and $f_{S}^{*}: 2^{S} \rightarrow \mathbf{R} \cup\{+\infty\}$ as follows:

$$
\begin{aligned}
& \mathcal{F}_{S}^{*}=\left\{R^{*}(B) \mid B \text { is a cobranching }\right\}, \\
& f_{S}^{*}(X)= \begin{cases}\min \left\{w(B) \mid B \text { is a cobranching, } R^{*}(B)=X\right\} & \text { if } X \in \mathcal{F}_{S}^{*}, \\
+\infty & \text { if } X \notin \mathcal{F}_{S}^{*}\end{cases}
\end{aligned}
$$

Similarly, in $D[T]$, define $\mathcal{F}_{T} \subseteq 2^{T}$ and $f_{T}: 2^{T} \rightarrow \mathbf{R} \cup\{+\infty\}$ by

$$
\begin{aligned}
& \mathcal{F}_{T}=\{R(B) \cap T \mid B \text { is a branching }\}, \\
& f_{T}(X)= \begin{cases}\min \{w(B) \mid B \text { is a branching, } R(B)=X\} & \text { if } X \in \mathcal{F}_{T}, \\
+\infty & \text { if } X \notin \mathcal{F}_{T} .\end{cases}
\end{aligned}
$$

By Theorem 6 , both $f_{S}^{*}$ and $f_{T}$ are $\mathrm{M}^{\natural}$-convex functions. Furthermore, the following proposition immediately follows from nonnegativity of $w$.

Proposition 7. The functions $f_{S}^{*}$ and $f_{T}$ are monotone non-increasing, that is, $f_{S}^{*}\left(X^{\prime}\right) \geq f_{S}^{*}(X)$ if $X^{\prime} \subseteq X$ and $f_{T}\left(X^{\prime}\right) \geq f_{T}(X)$ if $X^{\prime} \subseteq X$. 
Proof. Consider $f_{T}$. Let $X^{\prime} \subseteq X \subseteq V$ and suppose $X^{\prime} \in \operatorname{dom} f_{T}$. Let $B^{\prime} \subseteq A[T]$ be a branching in $D[T]$ attaining $f_{T}\left(X^{\prime}\right)$, that is, $R\left(B^{\prime}\right)=X^{\prime}$ and $w\left(B^{\prime}\right)=f_{T}\left(X^{\prime}\right)$. By removing arcs in $B^{\prime} \cap$ $\bigcup_{v \in X \backslash X^{\prime}} \delta^{-} v$ from $B^{\prime}$, we obtain another branching $B$ with $R(B)=X$. By nonnegativity of $w$, it holds that $w(B) \leq w\left(B^{\prime}\right)$. We consequently obtain that $f_{T}(X) \leq w(B) \leq w\left(B^{\prime}\right)=f_{T}\left(X^{\prime}\right)$.

A similar argument holds for $f_{S}^{*}$.

Now, the shortest $S-T$ bibranching problem is described as the following optimization problem:

$$
\begin{array}{lll}
(\mathrm{SBP}) & \text { minimize } & w(F)+f_{S}^{*}\left(\partial^{+} F\right)+f_{T}\left(\partial^{-} F\right) \\
& \text { subject to } & F \subseteq A[S, T] .
\end{array}
$$

In order to obtain a shortest $S$ - $T$ bibranching from an optimal solution $F_{0}$ for (SBP), it suffices to find a minimum-weight cobranching $B_{S}$ in $D[S]$ with $R^{*}\left(B_{S}\right)=\partial^{-} F_{0}$ and a minimum-weight branching $B_{T}$ in $D[T]$ with $R\left(B_{T}\right)=\partial^{+} F_{0}$, and then return $F_{0} \cup B_{S} \cup B_{T}$.

In the next subsection, we show a polynomial reduction of (SBP) to the valuated matroid intersection problem.

\subsection{Reduction to valuated matroid intersection}

We consider to transform $f_{S}^{*}$ and $f_{T}$ to valuated matroids whose ground sets correspond to $A[S, T]$. First, we extend the domain of $f_{S}^{*}$ to $\mathbf{Z}^{S}$. Define $g_{S}: \mathbf{Z}^{S} \rightarrow \mathbf{R} \cup\{+\infty\}$ by

$$
g_{S}(x)= \begin{cases}f_{S}^{*}\left(\operatorname{supp}^{+}(x)\right) & \text { if } x \in \mathbf{Z}_{+}^{S}, \\ +\infty & \text { if } x(v)<0 \text { for some } v \in S .\end{cases}
$$

Proposition 8. The function $g_{S}$ is an $M^{\natural}$-convex function.

Proof. For brievity, let us abbreviate $f_{S}^{*}$ and $g_{S}$ as $f$ and $g$, respectively. We prove that $g$ satisfies $\left(\mathrm{M}^{\natural}-\mathrm{EXC}\right)$.

Let $x, y \in \operatorname{dom} g$ and $u \in \operatorname{supp}^{+}(x-y)$.

Case $1(y(u) \geq 1)$. Note that $x(u) \geq 2$ holds. It is easy to observe that

$$
g\left(x-\chi_{u}\right)=g(x)=f\left(\operatorname{supp}^{+}(x)\right), \quad g\left(y+\chi_{u}\right)=g(y)=f\left(\operatorname{supp}^{+}(y)\right) .
$$

Thus, $g\left(x-\chi_{u}\right)+g\left(y+\chi_{u}\right)=g(x)+g(y)$ follows.

Case $2(x(u) \geq 2, y(u)=0)$. In this case, we have that $g\left(x-\chi_{u}\right)=g(x)$, and $g\left(y+\chi_{u}\right) \leq g(y)$ by Proposition 7. Thus, $g\left(x-\chi_{u}\right)+g\left(y+\chi_{u}\right) \leq g(x)+g(y)$ follows.

Case $3(x(u)=1, y(u)=0)$. Consider Property (M $\mathrm{M}^{\natural}$-EXC) of $f$. Let $X=\operatorname{supp}^{+}(x), Y=\operatorname{supp}^{+}(y)$. If $f(X \backslash\{u\})+f(Y \cup\{u\}) \leq f(X)+f(Y)$ holds, then $g\left(x-\chi_{u}\right)+g\left(y+\chi_{u}\right) \leq g(x)+(y)$ follows immediately.

Suppose $f((X \backslash\{u\}) \cup\{v\})+f((Y \cup\{u\}) \backslash\{v\}) \leq f(X)+f(Y)$ for some $v \in Y \backslash X$. Then, it follows that

$$
g\left(x-\chi_{u}+\chi_{v}\right)=f\left(\operatorname{supp}^{+}\left(x-\chi_{u}+\chi_{v}\right)\right)=f((X \backslash\{u\}) \cup\{v\}),
$$

and, from Proposition 7,

$$
g\left(y+\chi_{u}-\chi_{v}\right)=f\left(\operatorname{supp}^{+}\left(y+\chi_{u}-\chi_{v}\right)\right) \leq f((Y \cup\{u\}) \backslash\{v\}) .
$$

Thus, we have that $g\left(x-\chi_{u}+\chi_{v}\right)+g\left(y+\chi_{u}-\chi_{v}\right) \leq g(x)+g(y)$. 
We then transform $g_{S}$ so that its domain could be associated to $A[S, T]$. Define $h_{S}: \mathbf{Z}^{A[S, T]} \rightarrow$ $\mathbf{R} \cup\{+\infty\}$ by

$$
h_{S}(x)= \begin{cases}g_{S}\left(x^{\prime}\right) & \text { if } x \in\{0,1\}^{A[S, T]} \\ +\infty & \text { otherwise }\end{cases}
$$

where $x^{\prime} \in \mathbf{Z}^{S}$ is a vector defined by

$$
x^{\prime}(u)=\sum_{a \in \delta^{+} u} x(a) \quad \text { for each } u \in S .
$$

It is straightforward to see that $h_{S}$ is obtained from $g_{S}$ by transformation through the following network:

- The digraph is $(N, E)$, where $N=S \cup A[S, T]$ and $E=\left\{u a \mid u \in S, a \in A[S, T] \cap \delta^{+} u\right\}$. The entrance set is $S$ and the exit set is $A[S, T]$.

- $\underline{c}(e)=0, \bar{c}(e)=1$, and $\gamma_{e} \equiv 0$ for every $e \in E$.

By Theorem 4, we have that $h_{S}$ is an $\mathrm{M}^{\natural}$-convex function.

We further transform the $\mathrm{M}^{\natural}$-convex function $h_{S}$ to an M-convex function. Let $a_{0}$ be an element distinct from $A[S, T]$, and let $\tilde{A}=\left\{a_{0}\right\} \cup A[S, T]$. Let $\alpha \in \mathbf{Z}_{+}$be a sufficiently large integer ${ }^{1}$. Define $\tilde{h}_{S}: \mathbf{Z}^{\tilde{A}} \rightarrow \mathbf{R} \cup\{+\infty\}$ by

$$
\tilde{h}_{S}\left(x_{0}, x\right)=\left\{\begin{array}{ll}
h_{S}^{\prime}(x) & \text { if } x_{0}=\alpha-x(A[S, T]), \\
+\infty & \text { otherwise }
\end{array} \quad\left(x_{0} \in \mathbf{Z}, x \in \mathbf{Z}^{A[S, T]}\right) .\right.
$$

By Theorem 3, $\tilde{h}_{S}$ is an M-convex function.

Let $U$ be a set of elements disjoint from $A[S, T]$ with size $\alpha$ and denote $W=U \cup A_{W}$, where $A_{W}$ is a copy of $A[S, T]$. An element in $A_{W}$ corresponding to $a \in A[S, T]$ is denoted by $a_{W}$. Define $h^{+}: 2^{W} \rightarrow \mathbf{R} \cup\{+\infty\}$ by

$$
h^{+}(X)= \begin{cases}\tilde{h}_{S}\left(|X \cap U|, \chi_{X \cap A_{W}}\right) & \text { if }|X|=\alpha, \\ +\infty & \text { otherwise. }\end{cases}
$$

It is easy to observe that $h^{+}$is obtained from $\tilde{h}_{S}$ by transformation through the following network and identifying $X \subseteq V$ as $\chi_{X} \in\{0,1\}^{V}$ :

- The digraph is $(N, E)$, where $N=\tilde{A} \cup W$ and $E=\left\{a_{0} u \mid u \in U\right\} \cup\left\{a a_{W} \mid a \in A[S, T]\right\}$. The entrance set is $\tilde{A}$ and the exit set is $W$.

- $\underline{c}(e)=0, \bar{c}(e)=1$, and $\gamma_{e} \equiv 0$ for every $e \in E$.

Thus, by Theorem $4, \omega^{+}=-h^{+}$is a valuated matroid. We also obtain a valuated matroid $\omega^{-}$: $2^{W} \rightarrow \mathbf{R} \cup\{-\infty\}$ from $f_{T}$ by similar transformation.

\footnotetext{
${ }^{1}$ It suffices that $\alpha \geq|A[S, T]|-\min \left\{\min \left\{|X| \mid X \in \operatorname{dom} f_{S}^{*}\right\}, \min \left\{|X| \mid X \in \operatorname{dom} f_{T}\right\}\right\}$.
} 
We are now ready to define an instance of the valuated matroid intersection problem. The ground set is $W=U \cup A[S, T]$ and define a weight vector $\tilde{w} \in \mathbf{R}^{W}$ by

$$
\tilde{w}(v)= \begin{cases}0 & \text { if } v \in U, \\ -w(a) & \text { if } v \in A[S, T] .\end{cases}
$$

Now, we have constructed an instance $\left(W, \tilde{w}, \omega^{+}, \omega^{-}\right)$of the valuated matroid intersection problem. It immediately follows that, for an optimal solution $X$ for $\left(W, \tilde{w}, \omega^{+}, \omega^{-}\right)$, the set of arcs in $A[S, T]$ corresponding to $X \cap A_{W}[S, T]$ is an optimal solution for (SBP).

\section{Concluding remarks}

We have shown that the shortest $S-T$ bibranching problem falls in the framework of the valuated matroid intersection problem. Our argument begins with the fact that weighted branchings determine a valuation on a generalized matroid (Theorems 5 and 6), and then make use of theory of a more general framework of M-convex functions. That is, we transformed the valuation to an M-convex function, and then applied the operation of transformation through a network, in order to make the two valuations on $\{0,1\}^{S}$ and $\{0,1\}^{T}$ have the same ground set.

Let us mention the shortest $S$ - $T$ bibranching algorithms obtained from this reduction. By applying algorithms for the valuated matroid intersection problem [13] to the instance $\left(W, \tilde{w}, \omega^{+}, \omega^{-}\right)$ constructed in Section 3.2, we obtain new combinatorial algorithms for the shortest $S$ - $T$ bibranching problem. The algorithms in [13] essentially resemble to classical combinatorial matroid intersection algorithms, and we need a value oracle for $\omega^{+}$or $\omega^{-}$in constructing every exchangeability arc in auxiliary graphs. In our case, this value oracle is exactly computing $f(X)$ in $(6)$ or $f^{*}(X)$ in $(7)$, which can be performed efficiently by calling a minimum-weight arborescence algorithm.

We conclude this paper by mentioning one more application of Theorem 6 . In a recent paper of Shioura [21], the following problem is considered.

\section{The $k$-budgeted $\mathbf{M}^{\natural}$-concave maximization problem}

Input: - an $\mathrm{M}^{\natural}$-concave function $f: 2^{V} \rightarrow \mathbf{R} \cup\{-\infty\}$, - cost vectors $\gamma_{i}: V \rightarrow \mathbf{R}_{+}$and budgets $b_{i} \in \mathbf{R}_{+}(i=1, \ldots, k)$.

Objective: maximize $f(X)$ subject to $\gamma_{i}(X) \leq b_{i}$ for each $i=1, \ldots, k$.

For the $k$-budgeted $\mathrm{M}^{\natural}$-concave maximization problem, Shioura [21] presented a PTAS. We can consider one kind of the network design problem which falls in the framework of the $k$-budgeted $\mathrm{M}^{\natural}$-concave maximization problem.

Suppose that we are given a weighted digraph $(D, w)$ of $D=(V, A)$ and $w \in \mathbf{R}_{+}^{A}$. We should determine $X \subseteq V$, where to open facilities, and construct a subgraph of $D$ in which every vertex in $V \backslash X$ reaches some facility in $X$.

In opening facilities, we have $k$ budget-constraints, which are represented as linear constraints $\gamma_{i}(X) \leq b_{i}(i=1, \ldots, k)$. Connecting vertices by an arc $a \in A$ costs $w(a)$, and thus construction of a subgraph $(V, B)$ costs $w(B)$. Our objective is to find $X \subseteq V$ and $B \subseteq A$ which satisfy the budget constraints and achieve the minimum connection cost.

If $X$ is given, a minimal subgraph $(V, B)$ achieving the connection from $V \backslash X$ to $X$ is exactly a cobranching $B$ with $R^{*}(B)=X$, and thus the connection cost is represented by $f^{*}$ in $(7)$. By 
Theorem $6,-f^{*}$ is an $\mathrm{M}^{\natural}$-concave function, and thus minimizing $f^{*}(X)$ subject to $\gamma_{i}(X) \leq b_{i}$ $(i=1, \ldots, k)$ is a special case of the $k$-budgeted $\mathrm{M}^{\natural}$-concave maximization problem. In this special case, again the value oracle is computing $f^{*}(X)$, which can be done efficiently, and thus the PTAS in [21] can be applied.

\section{Acknowledgements}

The author is grateful to Satoru Iwata for discussion on this topic. This work was supported by Grant-in-Aid for Young Scientists from the Ministry of Education, Culture, Sports, Science and Technology of Japan.

\section{References}

[1] A.W.M. Dress and W. Wenzel: Valuated matroid: A new look at the greedy algorithm, Applied Mathematics Letters, 3 (1990), 33-35.

[2] A.W.M. Dress and W. Wenzel: Valuated matroids, Advances in Mathematics, 93 (1992), 214250.

[3] J. Edmonds and R.M. Karp: Theoretical improvements in algorithmic efficiency for network flow problems, in R. Guy, H. Hanani, N. Sauer and J. Schönheim, eds., Combinatorial Structures and Their Applications (Proceedings Calgary International Conference on Combinatorial Structures and Their Applications, Calgary, 1969), 1970, 93-96.

[4] A. Frank: A weighted matroid intersection algorithm, Journal of Algorithms, 2 (1981), 328336.

[5] A. Frank: Generalized polymatroids, in A. Hajnal, L. Lovász and V.T. Sós, eds., Finite and Infinite Sets, vol. I (Proceedings of the Sixth Hungarian Combinatorial Colloquium, Eger, 1981), 1984, 285-294.

[6] S. Fujishige: Submodular Functions and Optimization, Annals of Discrete Mathematics, 58, Elsevier, Amsterdam, second edition, 2005.

[7] H.N. Gabow, Z. Galil, T. Spencer and R.E. Tarjan: Efficient algorithms for finding minimum spanning trees in undirected and directed graphs, Combinatorica, 6 (1986), 109-122.

[8] M. Iri and N. Tomizawa: An algorithm for finding an optimal "independent assignment", Journal of the Operations Research Society of Japan, 19 (1976), 32-57.

[9] J. Keijsper and R. Pendavingh: An efficient algorithm for minimum-weight bibranching, Journal of Combinatorial Theory, Series B, 73 (1998), 130-145.

[10] E.L. Lawler: Matroid intersection algorithms, Mathematical Programming, 9 (1975), 31-56.

[11] K. Murota: Convexity and Steinitz's exchange property, Advances in Mathematics, 125 (1996), $272-331$.

[12] K. Murota: Valuated matroid intersection I: Optimality criteria, SIAM Journal on Discrete Mathematics, 9 (1996), 545-561. 
[13] K. Murota: Valuated matroid intersection II: Algorithms, SIAM Journal on Discrete Mathematics, 9 (1996), 562-576.

[14] K. Murota: Discrete Convex Analysis, Society for Industrial and Applied Mathematics, Philadelphia, 2003.

[15] K. Murota: Matrices and Matroids for Systems Analysis, Springer-Verlag, Berlin, softcover edition, 2010.

[16] K. Murota and A. Shioura: M-convex function on generalized polymatroid, Mathematics of Oprations Research, 24 (1999), 95-105.

[17] A. Schrijver: Min-max relations for directed graphs, Annals of Discrete Mathematics, 16 (1982), 261-280.

[18] A. Schrijver: Total dual integrality of matching forest constraints, Combinatorica, 20 (2000), $575-588$.

[19] A. Schrijver: Combinatorial Optimization-Polyhedra and Efficiency, Springer-Verlag, Heidelberg, 2003.

[20] A. Shioura: A constructive proof for the induction of M-convex functions through networks, Discrete Applied Mathematics, 82 (1998), 271-278.

[21] A. Shioura: Polynomial-time approximation schemes for maximizing gross substitutes utility under budget constraints, in C. Demetrescu and M.M. Halldórsson, eds., Proceedings of the 19th Annual European Symposium on Algorithms, LNCS 6942, Springer-Verlag, 2011, 1-12.

[22] K. Takazawa: Optimal matching forests and valuated delta-matroids, in O. Günlük and G.J. Woeginger, eds., Integer Programming and Combinatorial Optimization: Proceedings of the 15th International IPCO Conference, LNCS 6655, Springer-Verlag, 2011, 404-416.

[23] É. Tardos: Generalized matroids and supermodular colourings, in L. Lovász and A. Recski, eds., Matroid Theory (Proceedings Colloquium on Matroid Theory, Szeged, 1982), 1985, 359382.

[24] N. Tomizawa: On some techniques useful for solution of transportation network problems, Networks, 1 (1971), 173-194. 